\title{
Activation During Observed Parent-Child Interactions with Anxious Youths: A Pilot Study
}

\author{
Araceli Gonzalez • Phoebe S. Moore • Abbe M. Garcia • \\ Margo Thienemann $\cdot$ Lynne Huffman
}

Published online: 21 January 2011

(C) The Author(s) 2011. This article is published with open access at Springerlink.com

\begin{abstract}
Parent-child interaction paradigms are often used to observe dysfunctional family processes; however, the influence of such tasks on a participant's level of activation remain unclear. The aim of this pilot project is to explore the stimulus value of interaction paradigms that have been commonly used in child anxiety research. Twenty-nine parent-child dyads with clinically anxious $(n=16)$ and nonanxious $(n=13)$ youths engaged in a series of tasks (threat and non-threat) used in previous studies of parenting and youth anxiety. Heart rate (HR) data, as an indicator of physiological activation, were collected across tasks, and participants rated the perceived representativeness of their interactions in the laboratory to their usual behavior at home. Significant HR changes were observed for both parent and child. Change in child HR from baseline to nonthreat task was smaller than change in HR from baseline to threat tasks. Change in parent HR from baseline to ambiguous situations tasks was smaller than changes from baseline to other threat tasks. Differences in HR change
\end{abstract}

A. Gonzalez $(\bowtie)$

Joint Doctoral Program in Clinical Psychology,

San Diego State University / University of California, San Diego,

San Diego, CA, USA

e-mail: arg001@ucsd.edu

P. S. Moore

University of Massachusetts Medical School and UMass Memorial Medical Center,

Worcester, MA, USA

\section{A. M. Garcia}

Brown University Medical Center,

Providence, RI, USA

M. Thienemann $\cdot$ L. Huffman

Stanford University Medical Center,

Stanford, CA, USA between anxious and non-anxious children were explored. Participants rated laboratory interactions as similar to those experienced in the home. Results suggest that presumably emotionally-charged discussion tasks may produce increased activation compared to tasks that were designed to be more neutral. Implications for future research and limitations are discussed.

Keywords Child anxiety · Parent-child interactions . Behavioral observation

Theorists in the field of anxiety research posit an important role for parenting behavior in the development and maintenance of child anxiety disorders (e.g., Chorpita et al. 1998; Ginsburg and Schlossberg 2002; Manassis and Bradley 1994; Rubin and Mills 1991; Wood et al. 2003). Dysfunctional behavioral patterns may emerge between parent and child, which may then serve to create, sustain, or exacerbate anxiety in the child (Dadds et al. 1996; Hudson and Rapee 2001; Rapee 1997), and there is increasing evidence for reciprocal models of anxiety development and maintenance (Hudson et al. 2009; Silverman et al. 2009).

There is a growing consensus among researchers in the area of child anxiety that observational methodology is superior to self-, parent-, and clinician-report for assessing dysfunctional parent and child behaviors that may trigger or maintain child anxiety disorder (e.g., Hawes and Dadds 2006; McLeod et al. 2007; Wood et al., 2003). In the field of anxiety research, it is generally believed that the use of observational paradigms and collection of behavioral samples can circumvent problems associated with self-report measures, including interpretative and recall biases (e.g., Costa and Weems 2005; Krain and Kendall 2000; Vasey et al. 1995) and social desirability (Kendall and Chansky 1991), thus providing a more useful and valid representation 
of important parent-child processes. Empirical support for the use of observational methods has also emerged. A recent meta-analysis of the association between parenting and child anxiety reported a medium effect size $(r=0.28)$ of parenting in studies when observational methods were used, an effect significantly higher than those obtained from self-report $(r=0.20)$ or interview $(r=0.15)$ measures of parenting (McLeod et al. 2007). Together, these findings support the notion that the observed behavior approach to assessment may be a uniquely powerful tool for investigating the association between anxiety and family relationships.

Although observational paradigms have garnered both theoretical and empirical support as a best-practice model for the assessment of parent-child interaction, measurement of dyadic behavioral patterns presents conceptual and logistical challenges to research (e.g., quantification of behaviors, reliability of coding) and some aspects of this promising methodology have not been well evaluated. For example, it is likely that the different types of interaction tasks can produce varying levels of emotional activation and thereby influence the behavioral styles displayed by research subjects. Indeed, there is emerging evidence that not all interaction tasks are created equal. One examination of interactions between anxious mothers and their children provided evidence that observational research may elicit parenting behaviors at different frequencies depending on the nature of the interaction task (e.g., structured vs. unstructured; Ginsburg et al. 2006). Further, a recent meta-analysis of child and parent anxiety reported that structured discussion and performance tasks yielded higher effect sizes than unstructured tasks in assessing parental control (van der Bruggen et al. 2008). Thus, it appears that examination of the stimulus value of commonly used interaction tasks could enhance our current understanding of how tasks work and may help guide researchers with appropriate task selection. To provide a context for the current investigation regarding the stimulus value of commonly utilized family interaction paradigms, we briefly describe tasks that have been commonly used in the pediatric anxiety literature.

\section{Types of Interaction Tasks Currently Employed in Pediatric Anxiety Research}

Various paradigms have been used to assess parenting behaviors in an observational format. Frequently, parents and children are asked to engage in discussion or complete a task together, usually for a specified duration. For an overview of relevant findings from studies, see Ginsburg et al. 2004b; McLeod et al., 2007; van der Bruggen et al. 2008; and Wood et al. 2003. Below, we briefly describe four major types of tasks employed in recent research of parent-child interactions in families with an anxious parent or child:
Ambiguous Situations

(e.g., Barrett et al. 1996, 2002; Shortt et al. 2001; Varela et al. 2004). Variations of this task have often been utilized. Parent and child are given scenarios, typically related to potential physical or social threat. For example, dyads may be prompted with "You/your child sees a group of students from another class playing a great game. You/your child walks over and wants to join in, and hears the children laughing - What do you think is happening and what would your child do?" (e.g., Barrett et al. 2002, p. 600). The dyad is tasked with interpreting the scenario and determining an appropriate response. The degree of threat interpreted in the situation and the amount of avoidance employed in the selected response are often quantified in these tasks.

\section{Revealed Differences}

(Conflict and Anxiety conversations; Challacombe and Salkovskis 2009; McCarty et al. 2004; Moore et al. 2004; Siqueland et al. 1996; Whaley et al. 1999). Parent and child have two conversations, typically timed, about a conflict and/or something that is anxiety-provoking to the youth. For the conflict task, some investigators have asked the dyad to discuss a "hot topic" chosen by the mother (e.g., Siqueland et al. 1996) or a conflictual topic agreed upon by both dyad members (e.g., Moore et al. 2004; Whaley et al. 1999) and asked to attempt to reach an agreement or resolution. Presumably, these tasks are aimed at provoking stressful interactions in the dyad. When coding these tasks, investigators often use Likert-type scales to rate the frequency of or the degree to which certain pre-defined parenting behaviors are displayed (e.g., autonomy granting, control, warmth).

\section{Challenge/Cognitive Tasks}

(e.g., unsolvable anagrams/tangrams; Buckley and Woodruff-Borden 2006; Hudson and Rapee 2001; Woodruff-Borden et al. 2002; origami challenge task; Greco and Morris 2002). In these tasks, the child is presented with a challenging task (e.g., tangrams - putting smaller geometric pieces to form larger shapes; Hudson and Rapee 2001; unsolvable word puzzles; Buckley and Woodruff-Borden 2006), with little or no likelihood of success. The parent is typically instructed not to intervene unless the child requires help, however, some investigators instruct parents that it is OK to help (e.g., Buckley and Woodruff-Borden 2006). These situations allow for the observation of the child's reaction to stressful situations and the parent's behavioral reaction (e.g., overcontrol) to the child in an unsuccessful situation. Latency to parental response is often measured along with behavioral ratings, 
and global ratings of specific parenting dimensions and behaviors are generated.

\section{Other Tasks}

(e.g., preparing a speech; Buckley and Woodruff-Borden 2006; Cobham et al. 1999; Gar and Hudson 2008; Hudson et al. 2009; Woodruff-Borden et al. 2002; Etch-a-sketch; Ginsburg et al. 2004a; Ideal person task; Whaley et al. 1999; Moore et al. 2004; Grocery Store Game; Dumas et al. 1995; Risk Room; Turner et al. 2003). The majority of these tasks are aimed at challenging the dyad in some manner and raising stress levels - e.g., the parent must help the (sometimes socially anxious) child prepare a speech, typically on a topic of the child's choosing (e.g., Gar and Hudson 2008); the parent and child must work together to solve a difficult puzzle or create a piece of artwork (copy designs onto an Etch-A-Sketch toy with each dyad member controlling one of the two knobs; Ginsburg et al. 2004a). As with other types of tasks, investigators often attempt to quantify the occurrence of parenting behaviors (e.g., providing unsolicited help) or the expression of negative affect (e.g., Woodruff-Borden et al. 2002).

Rationale for Examining the Stimulus Value of Interaction Tasks

Researchers who utilize observational paradigms seldom discuss assumptions underlying the use of such tasks (Gardner 2000) but potential intentions can be inferred from the task design and content. Typically, parent-child dyads are not asked to discuss the weather or happy memories of family harmony, but instead are asked to engage in discussion or tasks that may produce dysfunctional behaviors - whether discussing an area of conflict, preparing a speech, or working on an unsolvable puzzle. It seems, then, that a potential goal of these tasks may be to provoke disagreement, anxiety, or frustration in order to elicit the dysfunctional behaviors hypothesized to characterize problematic interactions. Theoretically, this makes sense, as the overprotective, rejecting, or catastrophizing parenting behaviors that often are identified as risk or maintenance factors for child anxiety disorder may emerge only when the child or parent is anxious or otherwise emotionally aroused.

It is reasonable to suspect that dysfunctional interaction patterns would coincide with excitable emotions (e.g., anger, distress) and that physiological activation would ensue. However, the degree to which such arousal actually occurs during the interaction task has not been evaluated in the extant research. If arousal varies by task type, this may provide useful hypotheses about why some tasks may be more likely to produce dysfunctional behaviors than others.
Further, examination of potential physiological arousal may be useful in elucidating whether the tasks produce nonobservable features of anxiety that would not be captured in behavioral ratings. For example, Siqueland and colleagues (1996) noted that a child's expression of avoidance or submission in response to a controlling statement of the parent may not be captured in a behavioral coding system. Thus, activation may be a useful avenue by which to examine unobservable individual processes that occur during family interactions. Examination of the stimulus value of the tasks, independent of the observed behaviors associated with them, may be informative, and using arousal as an indicator of emotional reactivity seems an important step in understanding the stimulus value of tasks commonly used in pediatric anxiety research.

Another important consideration in this area of research is whether behaviors displayed during observational paradigms provide information regarding behavior patterns in "real-life" family situations. The argument that families may be reacting atypically to a novel or artificial setting is certainly valid. These concerns have been examined in studies with younger children with disruptive behaviors, and this research indicates that observation of behavior in a laboratory setting may be useful if the goal is to elicit behaviors of interest (see Gardner 2000). To date, this point has not been directly examined with anxious families. Although this question cannot be conclusively addressed through participant report, information regarding participants' subjective experience may be a useful first step towards examining the representativeness of their interactions. If interactions are perceived as unnatural or unrepresentative, then this may be a useful indicator of the ecological validity of such paradigms.

\section{Present Study}

As with any assessment tool, the properties of observation paradigms warrant examination, and insight into the stimulus value of commonly used tasks may provide useful information about what the tasks are accomplishing with participants. Surprisingly, little is known about the potential stimulus value of some of these commonly used observation paradigms in child anxiety research. The aim of this pilot investigation is to enhance the current literature on this methodology by assessing the experiences of dyads engaged in commonly used interaction tasks. Specifically, the focus of this study pertains to the methods used to assess parent-child interaction patterns rather than the relations of behaviors to child outcome. The primary goal of this study is to determine whether differences in level of emotional arousal, as indicated by changes in heart rate from a baseline level, emerge across various tasks. A second, exploratory aim is to examine whether participants' experiences of these tasks echo the 
experience of interacting in a "real life" context by exploring the perceived representativeness of tasks that occur in the research office setting.

We used a pilot sample of parent-child dyads with anxious and non-anxious youth to provide an initial exploration of the activating value of two of these commonly used observational paradigms (revealed differences and ambiguous situations tasks). We selected tasks that had been previously used in studies that produced significant group differences in dysfunctional behaviors and that easily allow participants to sit in a chair without requiring movement. To evaluate the hypothesis that tasks produce activation and arousal, heart rate (HR) data were gathered for both dyad members throughout the experiment as they engaged in a series of interaction tasks. Copious data suggest that anxious states are associated with increased HR (Beidel 1991; Weems et al. 2005) and that HR may be more strongly associated to selfreported anxiety symptoms than other measures of arousal (e.g., skin conductance; Weems et al. 2005). Therefore, participant HR data were collected as an indicator of psychophysiological arousal during interaction tasks aimed at increasing anxiety or emotional arousal in the dyad, as well as during comparison tasks designed to be nonthreatening. In addition, we conducted preliminary followup analyses to assess whether patterns of activation varied by child anxiety status. To evaluate parent and child perceived representativeness of interactions, participants were asked, upon completion of the interaction tasks, to provide ratings of how similar their laboratory behavior was to their usual behavior at home. This represents the first study to explore this issue in an anxious pediatric population.

We hypothesized that, on a within-subject basis, revealed differences and ambiguous situation parent-child interaction tasks would be associated with greater HR change from baseline than a low-demand comparison task that did not involve anxiety or conflict-related content. Furthermore, we predicted that parents and children would rate their behavior in the laboratory situation as similar to their behavior at home. Additionally, in exploratory analyses, we sought to evaluate whether changes in HR across tasks differed for anxious and non-anxious children. In order to better understand each group's response to the interaction task types, we also planned to conduct within-group analyses examining patterns of HR change within the group of anxious children and, separately, within the group of non-anxious children.

\section{Method}

The current study was approved by the Institutional Review Board at the study institution. All parents and youths provided signed informed consent and assent, respectively, after reviewing the consent form in detail with a Ph.D.-level member of the research team at the beginning of the first meeting with researchers.

\section{Participants}

A total of 29 dyads, consisting of 28 mothers and 1 father (Mean age $=45.74$ years, $S D=5.48$ ) and their 8- to 16-yearold children $(M=11.97, S D=2.68 ; 41.4 \%$ male $)$, participated in this study. Participants were primarily Caucasian families $(80.3 \%)$ of middle-high socioeconomic status, representative of the surrounding community in northern California (Mean annual household income $>\$ 100,000$ ). Each participating parent was the primary caregiver of his/ her child. Dyads were recruited based on child anxiety status. Anxious children were recruited through referrals from clinicians at a university-based anxiety disorders clinic in northern California. Non-anxious children were recruited through flyers and announcements in the general community. Two children who were recruited as nonanxious comparisons met criteria for a clinically impairing anxiety disorder during the diagnostic evaluation and were thus included in the anxious child group. Characteristics of the two groups and the sample as a whole are summarized in Table 1.

Children with a clinically impairing anxiety disorder $(n=16 ; 55.2 \%)$ met criteria for primary diagnoses of social phobia $(n=5)$, generalized anxiety disorder $(n=4)$, obsessivecompulsive disorder $(n=2)$, specific phobia $(n=3)$, or separation anxiety $(n=2)$. Comorbidity with another anxiety disorder was common; the mean number of anxiety diagnoses in anxious children was $2.81(S D=1.72)$.

\section{Measures}

Diagnostic Assessment Child anxiety diagnoses were assessed using the Anxiety Disorders Interview Schedule for Children and Parents (ADIS-IV-P/C; Silverman and Albano 1996) during the first visit. Child and the parent were each interviewed about the child. The ADIS-IV-P/C is a semi-structured interview designed to generate diagnoses based on the integration of both parent and child responses. Reliability data on the child ADIS-IV have been found to be excellent (Silverman et al. 2001). Parent and child diagnostic interviews were conducted by a Ph.D.-level investigator. All diagnostic questions regarding the participating children were reviewed by a team of experienced psychologists, psychiatrists, and psychology graduate students who met in conference to arrive at consensus diagnoses. This consensus process involved a thorough review and discussion of all symptoms endorsed by the child or parent on the diagnostic interview. To establish reliability, a subset of a randomly selected $20 \%$ of the child interviews were co-rated using audiotapes; raters were in 
Table 1 Demographic and clinical characteristics of anxious and non-anxious youths and their parent

Differ by child anxiety status $p<.05$ level

complete agreement as to presence/absence of current and past anxiety diagnoses (kappa $=1.0)$.

\section{Procedures}

All behavioral and physiological data on dyadic interactions was collected during the second visit or during the latter part of the visit if completed in one visit.

\section{Psychophysiology}

Heart Rate (HR) The psychophysiology equipment used to collect heart rate (HR) was a James Long Co. integrated system. Data were converted to average beats per minute (bpm) for each epoch, and HR was collected on a secondby-second basis. During data collection, dyads were seated upright in chairs across from each other. Leads were attached and data acquisition checked. Mean HR was calculated for seven epochs of data, presumed to reflect different levels of activation, from parent and child: Baseline, Joke Task, Conflict Task, Anxiety Conversation, Tummyache Conversation, Schoolyard Conversation, and Recovery, as described below.

Movement Because HR is associated with level of individual movement, we attached motion sensors to the backs of each chair to account for potential differences in movement across task. Both chairs were wire framed and permitted a certain amount of bouncing by participants.

\section{Dyadic Interaction Tasks}

Each dyad completed the following interactions while HR and movement data were collected.

Baseline (2 $\mathrm{min}$ ) The examiner entered the room and sat in a chair $5 \mathrm{ft}$ from the dyad, facing them. Dyads were instructed, "For the next $2 \mathrm{~min}$, we will just sit quietly and try to relax." The examiner kept time using a digital timer and looked down at a clipboard during this period.
The purpose of this task was to collect a baseline HR level.

Joke Task (3-5 min) This task was designed as a nonthreat, non-emotionally charged, collaborative interaction task. Once the baseline was completed, the examiner handed a list of five jokes to the parent, including the answers, and a list of the jokes to the child, without the answers. Using standardized instructions, she instructed the mother to read the jokes, one at a time, to the child; the child was to try to guess the answers to the jokes. The dyad was further instructed to discuss the jokes and decide together which was the funniest joke and which was the least funny. The examiner left the room after providing instructions and returned when summoned by the dyad or after $5 \mathrm{~min}$; at this time, she asked the dyad for their decision on the jokes.

Conflict Conversation (5 min) The Conflict Task was modeled on the task used by several research groups (e.g., Gordon et al. 1989; Moore et al. 2004; Siqueland et al. 1996; Whaley et al. 1999). Prior to collection of behavioral and physiology data, parents and children were asked independently to generate lists of conflict topics for the conflict task. The examiner chose a topic on which parent and child agreed; if no topic was agreed upon, the examiner chose a parent-selected topic. The dyad was instructed to talk about the conflict topic for 5 min with an attempt to reach an agreement or solve the problem. The examiner left the room after giving the instructions for the task. After $5 \mathrm{~min}$, the interviewer re-entered the room and redirected the dyad toward the next task.

Anxiety Conversation (5 min) (Moore et al. 2004; Whaley et al. 1999). The dyad was asked to talk for $5 \mathrm{~min}$ about something that made the child anxious or worried. These were the only instructions given to the dyad. Again, the examiner left the room after giving the instructions for the task. After $5 \mathrm{~min}$, the interviewer re-entered the room and redirected the dyad toward the next task. The order of Conflict and Anxiety was counterbalanced to control for order effects within task type. 
Ambiguous Situations ( $\leq 5$ min) (Barrett et al. 1996, 2002; Shortt et al. 2001; Varela et al. 2004). The dyad was presented verbally with two individual "ambiguous situations," one detailing possible social threat ("[Child's name] is playing the schoolyard, and s/he notices a group of kids from another class playing a great game. As s/he walks over and wants to join in, s/he notices they are laughing") and the other detailing possible physical threat ("On the way to school, [Child's name] starts to get a stomachache"). The dyad was asked to discuss the situation and decide what is happening and what the child should do. The examiner left the room for each discussion and returned when summoned to ask the dyad what they had decided. Scenarios were presented one at a time, with discussion following, in a counterbalanced order. HR data for these two separate discussions were combined into one data collection epoch for two reasons. First, from a theoretical standpoint, both discussions pulled for the same parentchild behavioral process, 1) the interpretation of ambiguous stimulus and 2) the effect of discussion on the likelihood of a threat interpretation. Second, from a more pragmatic position, the conversations tended to be short (averaging approximately $2 \mathrm{~min}$ ) and thus combining the two conversations resulted in an epoch of comparable elapsed time to the other behavioral data collection epochs. There were no differences in average HR for the two ambiguous tasks.

Recovery $(2 \mathrm{~min})$ At the conclusion of the ambiguous situations tasks, dyad members were asked to sit quietly for $2 \mathrm{~min}$. As with the Baseline Task, the examiner was present and modeled sitting quietly with her eyes directed downward.

\section{Ratings of Perceived Representativeness}

Upon completion of interaction tasks, parent and child were each asked to rate how similar their experience in the laboratory was to their experience of their typical behavior at home. Each dyad member was asked to make a Likerttype rating ranging from 1 to $10(1=$ not at all similar, $10=$ extremely similar) in response to the question "How similar was your behavior here today with your parent/child to your usual behavior at home?" This rating was a later addition to the protocol and was administered after the fifth dyad was assessed. Therefore, only 24 dyads completed this rating (four anxious families and one non-anxious family did not receive this rating form).

\section{Data Analytic Plan}

Steps were taken to quantify physiological data. HR was averaged across epochs in beats per minute (bpm). Change scores were computed for the HR by subtracting the average value obtained during Baseline from the average HR value during the relevant epoch; analyses were conducted using these change scores.

While recent trends in the field have focused on the advantage of mixed models for analyzing repeated measures (e.g., Gueorguieva and Krystal 2004), this global approach tends to require larger sample sizes than was available in this pilot study. We instead utilized repeated measures analysis of variance, or rANOVA, which allows for direct, one-at-a-time hypothesis testing. Violations of the assumption of sphericity, when found, were corrected using the Huynh-Feldt correction (Keselman et al. 2001).

We examined correlations between youth age and heart rate change score for each epoch to determine if the relationship of age to HR change scores should be controlled in analyses. We did not find any significant correlations between HR and age for any task $(r=-.21-.09$, all $p>.05)$, therefore, given sample size and associated power, we did not include age as a covariate in analyses. Because there were significantly more anxious than nonanxious boys in this sample (Table 1), we did control for gender in analyses of child HR data. In addition, we conducted a preliminary test of whether movement, which can influence HR, differed across non-threat and threat tasks. When controlling for gender, the association between task and movement was not significant, $F(6,156)=1.50$, $p=.18$, and therefore movement was not included in subsequent analyses.

Our primary hypotheses were tested in the whole sample, examining patterns of HR change epoch-byepoch. To explore the question of whether anxious children differed from their non-anxious peers on HR change scores for the various tasks, the repeated measures model was tested with anxiety status as a covariate. As a follow-up exploratory analysis, we sought to explore whether withinsubject patterns of HR change across tasks varied between anxious and non-anxious children. Therefore, to evaluate within-subject differences between specific epochs, planned a priori follow-up pairwise contrasts were used for child data, comparing changes in HR from one task to the next for each youth anxiety status group. Given the sample size and associated power, our analyses are exploratory rather than conclusive and therefore alpha was set at .05 for all analyses.

\section{Results}

Demographics

There were significantly more boys in the anxious than non-anxious group, $\chi^{2}(1)=6.56, p=.01$; anxious and non- 
anxious children did not differ on any other demographic variables (Table 1). Parents of anxious children were older than parents of non-anxious children, $t_{27}=2.08, p=.047$; parents of anxious and non-anxious youths did not significantly differ on any other demographic or clinical variables. Table 2 provides mean raw HR for children and parents. There was no significant difference in HR at baseline between anxious and non-anxious children, $t_{28}=$ $1.09, p=.29$, nor between parents of anxious and nonanxious children. HR did not significantly differ between anxious and non-anxious youths on any task.

\section{Within-subject HR Change}

The HR patterns for children and parents are illustrated in Figs. 1 and 2, respectively. Visual inspection of these graphs indicates that HR varied across tasks. The HR change patterns are illustrated in Figs. 3 and 4, respectively. HR change scores for participants are reported in Table 3 for each epoch.

Within-subjects main effects were significant, demonstrating significant within-subject differences in HR change across the interaction tasks for parents, $F(4.00,112.00)=47.64$, $p<.001$, and children, $F(3.33,89.87)=3.30, p=.02$, (child analyses controlled for gender; Huynh-Feldt correction was made for both tests). For children, Joke Task HR change scores (i.e., average Joke Task HR minus average Baseline HR) were smaller than Conflict Task, Anxiety Task, and Ambiguous Situation HR change scores, suggesting that the Joke Task was less activating than the other tasks for the

Table 2 Mean HR by child anxiety status

\begin{tabular}{llllll}
\hline \multirow{2}{*}{ Task } & \multicolumn{2}{l}{ Child not anxious } & & \multicolumn{2}{l}{ Child anxious } \\
\cline { 2 - 3 } \cline { 6 - 7 } & $\mathrm{M}^{\mathrm{a}}$ & $\mathrm{SD}$ & & $\mathrm{M}$ & $\mathrm{SD}$ \\
\hline Children & & & & \\
Baseline & 79.39 & 6.87 & & 83.07 & 10.64 \\
Jokes & 84.06 & 4.82 & & 83.58 & 11.79 \\
Conflict & 84.34 & 8.79 & & 87.80 & 12.19 \\
Anxiety & 84.10 & 6.54 & & 87.34 & 12.47 \\
Ambiguous situations & 84.25 & 7.68 & & 87.09 & 12.51 \\
Recovery & 79.03 & 7.62 & & 82.42 & 11.73 \\
Parents & & & & \\
Baseline & 67.34 & 7.49 & & 69.55 & 10.16 \\
Joke & 71.15 & 7.64 & & 73.42 & 10.65 \\
Conflict & 69.36 & 7.52 & & 72.79 & 11.72 \\
Anxiety & 70.02 & 8.58 & & 73.32 & 11.55 \\
Ambiguous situations & 68.78 & 7.09 & 73.9 & 11.32 \\
Recovery & 64.74 & 7.60 & 69.49 & 10.80 \\
\hline
\end{tabular}

${ }^{\mathrm{a}} \mathrm{HR}$ difference was not statistically significant on any task entire group of children, $F(1,27)=7.55, p=.011$. There were no significant differences in HR change scores among the threat tasks. For parents, Joke Task HR change scores were similar to Conflict Task and Anxiety Task HR change scores. Parental HR change score during the Ambiguous Situations Task was significantly smaller than HR change score during the Revealed Differences Tasks, $F(1,28)=9.83, p=.004$, although still positive, indicating higher HR than Baseline HR, $t_{27}=3.27, p<.005$. On average, Recovery Task HR change scores for parents and children had a negative valence, indicating that Recovery Task HR was lower than Baseline HR.

Exploratory Analyses: Main Effect of Child Anxiety and Interaction of Child Anxiety with Task Type

We examined whether the influence of task type on HR differed by youth anxiety status (controlling for gender). Although the average HR at Baseline was higher for anxious versus non-anxious children, this difference did not reach statistical significance $\left(t_{28}=1.09, p=.09\right)$. The between-subjects main effect for anxious and non-anxious children was non-significant, $F(1,26)=0.82, p=.77$, indicating that across all tasks, HR change scores for anxious children were generally not larger than HR change scores for non-anxious children. The within-subjects by betweensubjects interaction effect of child anxiety status by task was also non-significant $F(3.52,91.61)=1.18, p=.32$, with Huynh-Feldt correction for sphericity, indicating that the two groups did not significantly differ across tasks.

\section{Exploratory Analyses: Within-Group Examination of HR Change Across Task Type}

We proceeded with our planned follow-up within-subjects contrasts to separately explore the pattern of HR change within the groups of anxious and non-anxious youths, respectively. Within the sample of anxious children, there were significantly larger HR change scores for the Conflict Task and Anxiety Task compared to the Joke Task, (controlling for gender) $F(1,14)=7.03, p=.02$. Change scores for Ambiguous Situations Task were not significantly larger or smaller than for Conflict Task and Anxiety Task, indicating that anxious children remained equally activated through the remainder of the threat tasks.

In contrast to the findings with the anxious youth sample, we did not find significant difference in HR change scores for Jokes Task compared to Anxiety and Conflict Tasks for non-anxious youths, $F(1,11)=0.71, p=.42$ (controlling for gender) and the similar magnitude in change scores suggests that Revealed Differences Tasks are not more activating than the Joke Task for non-anxious youths. There were no differences in change in movement across 
Fig. 1 Mean raw HR scores across tasks by child anxiety status

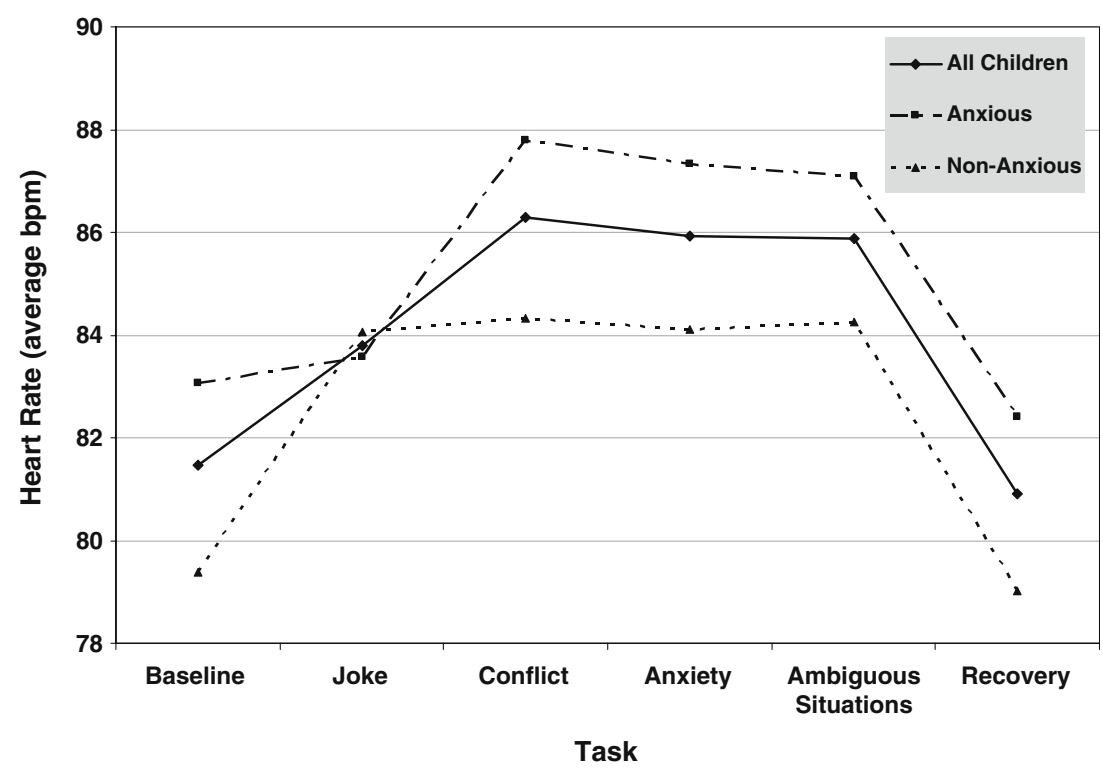

tasks between anxious and non-anxious youths, $F(3.55$, 92.21) $=0.93, p=.44$, suggesting that these results are not simply an artifact of anxious children being more animated during certain tasks.

There was no significant interaction between child anxiety status and within-parent HR change, indicating that the magnitude of HR changes for parents did not vary significantly by child anxiety status. Follow-up contrasts for parents were not planned a priori and were therefore not conducted.

\section{Perceived Representativeness Ratings}

Overall, both parents and children rated their behavior during interaction tasks as similar to their behavior in the home setting (range of mean ratings $=7-10$ ). Ratings between all parents $(M=8.08, S D=1.77)$ and all youths $(M=7.96, S D=1.65)$ did not significantly differ. Representativeness ratings for children did not vary by child anxiety status; however, parents of non-anxious children $(M=9.08$, $\mathrm{SD}=0.79)$ rated their behaviors as more representative of at-home interactions than did parents of anxious children $(M=7.08, S D=1.93), t_{22}=3.32, p<.005$.

\section{Discussion}

The aim of this project was gain a better understanding of the experiences of anxious dyads who engage in behavioral research paradigms. Specifically, we sought to assess HR, presumed to indicate emotional activation, and perceived
Fig. 2 Mean heart rate (bpm) of parents of anxious and nonanxious children

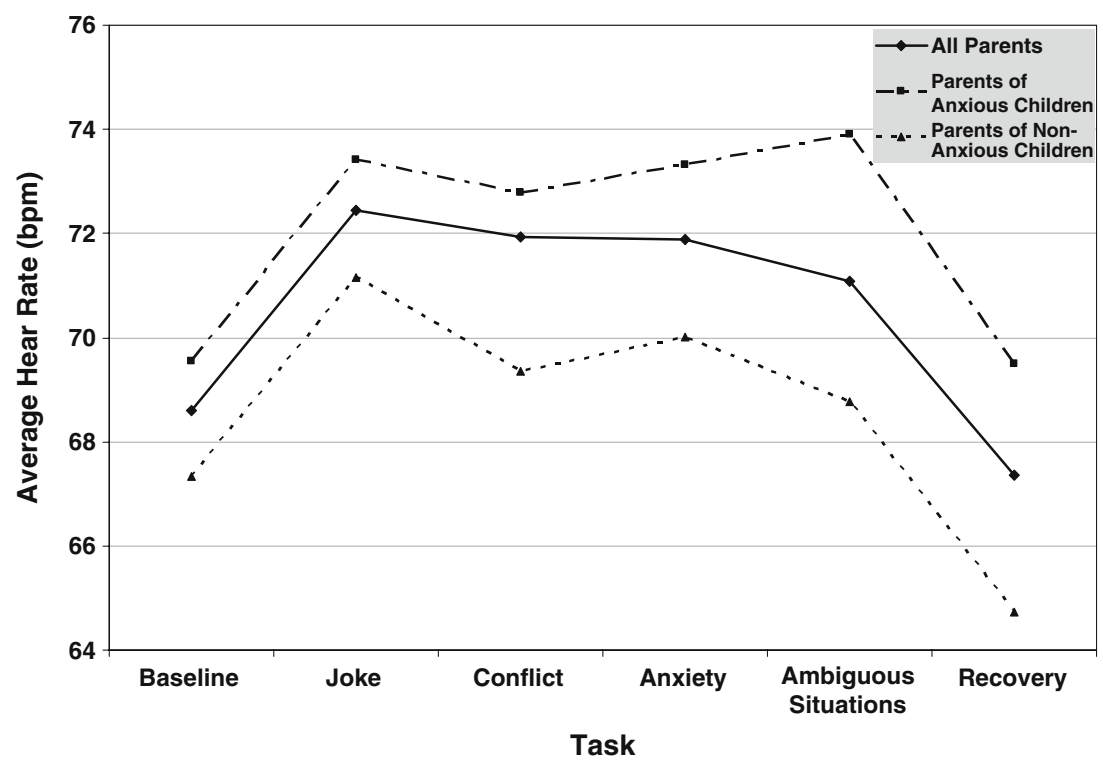


Fig. 3 Mean heart rate change score (change in bpm from baseline) of anxious and non-anxious children across task

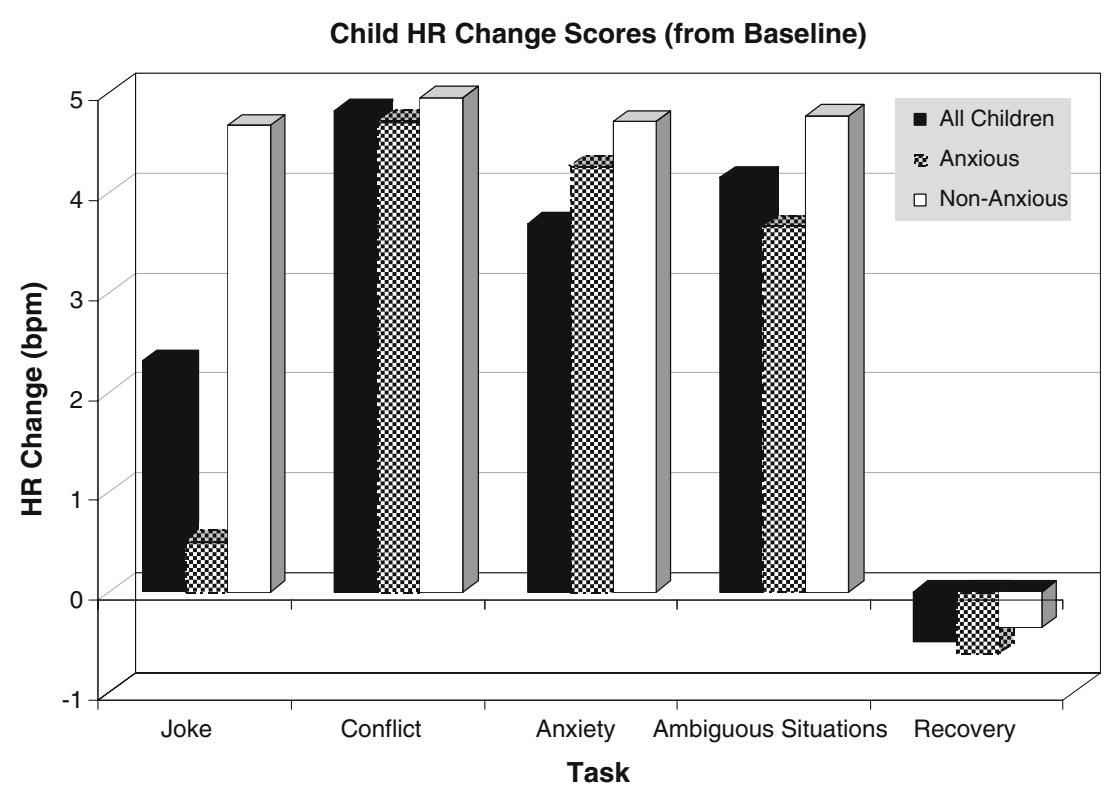

representativeness of behavior during interaction tasks commonly used in research on families affected by pediatric anxiety. This is among the first investigations to explore the stimulus value of these paradigms, and this information may be useful for the generation of testable hypotheses regarding the construct and ecological validity of behavioral paradigms that appear frequently in the extant literature base.

The tasks employed in this study were physiologically activating for both parents and children, as indicated by positive HR change scores during interaction tasks. However, parents and children showed different patterns of changes. Overall, children had relatively lower HR response to a non-threat task in which dyads rate jokes, whereas parents showed a lower response to discussion of ambiguous situations. Thus, the hypotheses that deliberately activating (i.e., threat) tasks (Revealed Differences and Ambiguous Situations) would be more activating than the low-demand (i.e., non-threat) comparison task (Joke Task) was supported in the child sample. This finding requires replication in larger samples.

We also explored whether within-subjects changes in HR across task differed for anxious and non-anxious youths. We did not detect a significant main effect of child anxiety status on HR across tasks, however, separate examination of each clinical group provides some interesting within-group data on how the series of tasks was experienced by children. Within the non-anxious group, all active tasks, both threat and nonthreat, were associated with the same size positive change from baseline. This suggests that the type of task may not have been relevant for the non-anxious group — interaction with the
Fig. 4 Mean heart rate change score (change in bpm from baseline) of parents of anxious and non-anxious children

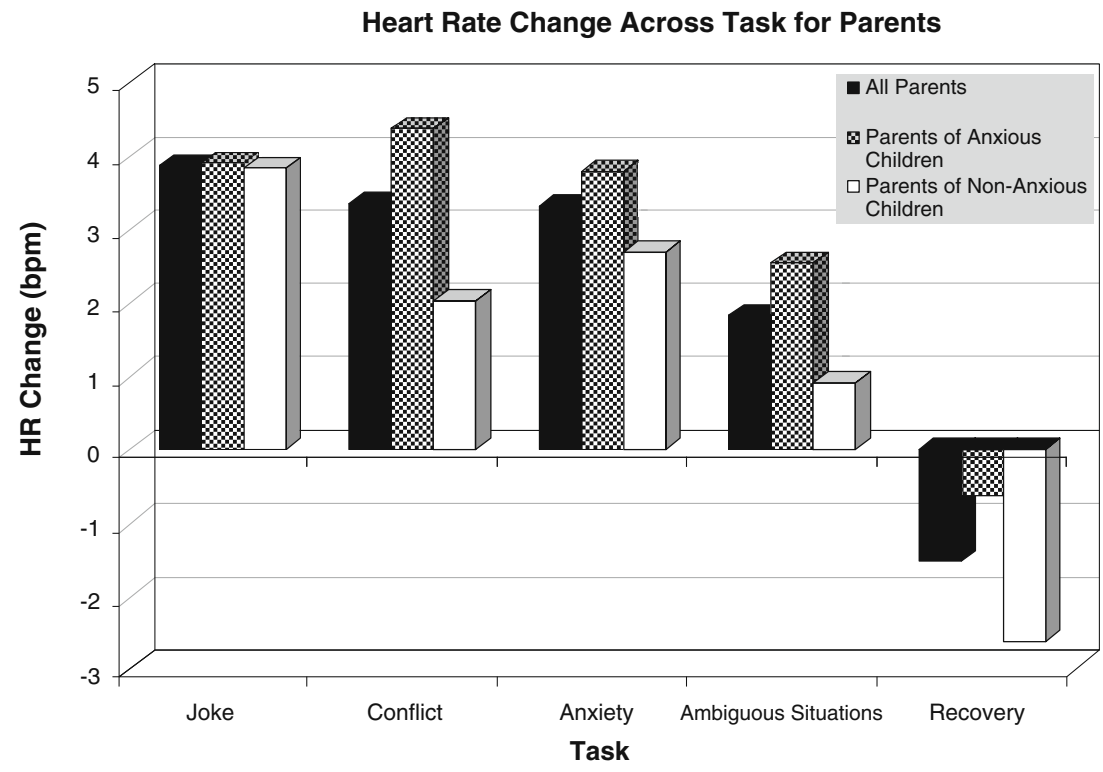


Table 3 Mean (SD) heart rate change score (change from baseline to task) by child anxiety status

\begin{tabular}{|c|c|c|c|c|c|c|}
\hline & Joke & Conflict & Anxiety & Ambiguous situations & Recovery & $F^{\mathrm{d}}$ \\
\hline \multicolumn{7}{|l|}{ Children } \\
\hline All & $2.39^{\mathrm{a}}(5.03)$ & $5.02^{\mathrm{b}}(3.99)$ & $4.69^{\mathrm{b}}(3.53)$ & $4.37^{\mathrm{b}}(4.31)$ & $-0.50^{\mathrm{c}}(3.29)$ & $15.57 * * *$ \\
\hline Anxious & $0.55^{\mathrm{a}}(4.30)$ & $5.07^{\mathrm{b}}(3.95)$ & $4.67^{\mathrm{b}}(3.98)$ & $4.06^{\mathrm{b}}(5.29)$ & $-0.62^{\mathrm{a}}(2.84)$ & $17.22 * * *$ \\
\hline Non-anxious & $4.67^{\mathrm{a}}(5.08)$ & $4.95^{\mathrm{a}}(4.20)$ & $4.71^{\mathrm{a}}(3.04)$ & $4.77^{\mathrm{a}}(2.82)$ & $-0.36^{\mathrm{b}}(3.88)$ & $5.13 * *$ \\
\hline \multicolumn{7}{|l|}{ Parents } \\
\hline All & $3.96^{\mathrm{a}}(2.48)$ & $3.37^{\mathrm{a}}(3.04)$ & $3.35^{\mathrm{a}}(3.00)$ & $1.88^{\mathrm{b}}(2.95)$ & $-1.52^{\mathrm{c}}(2.47)$ & $47.64 * * *$ \\
\hline Parents of anxious children & $4.08^{\mathrm{a}}(2.37)$ & $4.46^{\mathrm{a}}(3.46)$ & $3.91^{\mathrm{a}}(3.49)$ & $2.67^{\mathrm{b}}(3.20)$ & $-0.63^{\mathrm{c}}(2.60)$ & $31.93 * * *$ \\
\hline Parents of non-anxious children & $3.81^{\mathrm{a}}(2.69)$ & $2.02^{\mathrm{b}}(1.76)$ & $2.68^{\mathrm{b}}(2.19)$ & $0.90^{\mathrm{b}}(2.38)$ & $-2.60^{\mathrm{c}}(1.87)$ & $20.23 * * *$ \\
\hline
\end{tabular}

${ }^{\mathrm{d}} F$ for within-subjects analyses; significance adjusted for violations of sphericity via Huynh-Feldt correction

Items in a row with differing subscripts were significantly different at the $p<.05$ level

$* * p<.01, * * * p<.001$

parent, of any type, led to an increase in HR of about five bpm. This increase resolved at Recovery, wherein the children returned to Baseline HR levels.

Within the anxious group, a different pattern emerged. The HR change from Baseline to Jokes Task was significantly smaller than the HR change from Baseline to the three threat tasks. Visual inspection of the raw HR data suggests that HR at baseline was similar to HR at Joke Task (the change in average HR from Baseline to Joke Task was a clinically negligible $0.51 \mathrm{bpm}$ ). There followed a significant increase in HR change score once the threat tasks were introduced. This increase resolved at Recovery with children returning to Baseline HR levels. This pattern suggests the possibility of a specific response to the threat tasks within the anxious sample. In other words, it is possible that anxious children respond to the kinds of tasks used in observational research with a particularly robust increase in arousal that is different from their response to other, less-threatening tasks. This may provide support for the construct validity of the Revealed Differences and Ambiguous Situation task designs.

An additional exploratory aim of the present study was to probe parent and child perspectives about the "real-life" representativeness of laboratory interaction tasks. Results provide preliminary evidence that the subjective experience of parents and children of interactions in the laboratory setting are perceived as similar to those that occur in the home setting. While parents of both anxious and nonanxious children generally rated their observed behaviors as representative of those displayed in the home, parents of anxious children rated their behaviors as somewhat less representative. This detected difference may have implications for interpretation of findings produced from observational studies. Future research would benefit from evaluation of representativeness of behavior as well as exploration of how interactions at home may differ (e.g., Do parents feel that their children were more/less activated at home? Are parents of anxious children more likely to change their behavior in the laboratory setting?). It should be noted that this study assessed perceived representativeness and that inferences regarding actual representativeness would require independent measures of real-world interactions (e.g., in the home setting).

The hypothesis that non-threat and threat tasks would be associated with different HR arousal was not supported using parental HR. Recent studies have questioned the directionality of the influence of child anxiety (Hudson et al. 2009; Silverman et al. 2009); if reciprocal models of anxiety indicate that child anxiety may in turn elicit anxious parenting behaviors, and if anxious youths are experiencing activation during these tasks, results from the present study suggests that these tasks may still be acceptable for use with parents experiencing varying levels of anxiety.

To date, very little is known regarding the stimulus value of such interaction tasks. While it is not explicitly stated, one potential assumption is that the selected tasks are physiologically and emotionally activating. It is reasonable to suspect that tasks that are arousing may have behavioral correlates (e.g., dysfunctional behaviors). While it is possible that some parental behaviors, such as control, may function to prevent or reduce youth anxiety/distress and activation, an alternate hypothesis is that parents engage in those behaviors once youth arousal has been detected. It would be informative to conduct timesequenced coding of physiological activation in conjunction with behavioral data in order to determine which hypothesis may be more accurate in explaining associations between arousal and behaviors.

Findings from the present pilot study may provide an additional line of support for investigators seeking to utilize interaction tasks with anxious children and their parents. However, results must be viewed as preliminary and several limitations of this project warrant attention. The small sample size precluded the use of hierarchical analytic 
techniques, which may be appropriate for examining repeated emotional activation nested within dyads. Furthermore, exploratory analyses were underpowered for confirmatory hypothesis testing. It is possible that some relationships may have emerged as significant in larger samples, and our exploratory findings should be used for hypothesis generation only. In addition, this sample included primarily Caucasian, high-income families, and findings should be replicated with ethnically and socioeconomically diverse families.

We used change in HR from baseline to task as a proxy of emotional activation. A more comprehensive sampling of physiological data would enhance the specificity of findings. Because cardiac arousal is associated with a number of emotions, we are unable to specify whether the interaction tasks evoked anxiety or another emotion. Nevertheless, given the content of conversation tasks (e.g., conflict, anxiety), it is likely that physiological arousal was due to some distress rather than a positive emotion (e.g., excitement). It is possible that the baseline relaxation task was not entirely relaxing for those prone to anxiety because they were in a novel setting, and this may contribute to the observed lack of change in HR between Baseline and Joke Task for the anxious youths. While the difference in HR at baseline between anxious and nonanxious youths was not statistically significant, this alternative explanation warrants further examination with larger samples. By contrast, it is also possible that interaction tasks elicited other forms of negative affect or responses that may not have been captured using HR (e.g., avoidance). In future studies that aim to identify associations between activation and behaviors, it would be informative to code for indices of positive or negative affect in order to relate activation to specific aspects of parent and child emotion. In addition, the method with which the content of the discussions tasks was selected makes it difficult to determine whether activation was influenced by the process of initiating discussions (e.g., examiner providing a topic vs. agreeing on a topic in the moment). Our goal in this paper was to assess the stimulus value of commonly used tasks as they have been implemented previously. While we did not detect HR differences within the Revealed Differences Tasks within parent or youth participants, investigators may choose to alter the administration of these tasks in future studies to address this potential confound.

Because the order of tasks was not counterbalanced overall (only within task type), it is possible that timing, rather than type of tasks, contributed to the pattern of findings. For example, it is possible that parental HR for Revealed Differences Tasks was higher than during discussion of Ambiguous Situations because parents were aware that discussion of Ambiguous Situations was the last task. Further evidence is needed from counterbalanced designs. Future work may also benefit from inquiring about representativeness after each individual task rather than at the end for all tasks. Finally, while the current study aimed to examine frequently used interaction tasks, knowledge may be enhanced with investigation of more fear-provoking tasks that do not require dyads to sit still (e.g., a videotaped speech task).

Observer ratings of behaviors employed during interaction tasks are becoming the gold standard of methods used to better understand family interaction patterns. Pending replication with larger samples, present findings are promising in that they provide preliminary evidence that some commonly used family assessment tasks may indeed be having their hypothesized effect - to arouse emotion. This effect, which is relevant to the construct validity of this type of behavioral assessment, had not been previously evaluated. In addition, families endorse their interactions during these tasks as representative of those experienced in everyday life. While actual representativeness remains to be determined, these results provide a promising indication that laboratory tasks such as these may possess external validity with some samples.

Open Access This article is distributed under the terms of the Creative Commons Attribution Noncommercial License which permits any noncommercial use, distribution, and reproduction in any medium, provided the original author(s) and source are credited.

\section{References}

Barrett, P. M., Rapee, R. M., Dadds, M. M., \& Ryan, S. M. (1996). Family enhancement of cognitive style in anxious and aggressive children. Journal of Abnormal Child Psychology, 24, 187-203.

Barrett, P., Shortt, A., \& Healy, L. (2002). Do parent and child behaviours differentiate families whose children have obsessive-compulsive disorder from other clinic and non-clinic families? Journal of Child Psychology and Psychiatry, 43, 597-607.

Beidel, D. C. (1991). Determining the reliability of psychophysiological assessment in childhood anxiety. Journal of Anxiety Disorders, 5, 139-150.

Buckley, A. F., \& Woodruff-Borden, J. (2006). Maternal modeling of coping: relation to child anxiety. Child \& Family Behavior Therapy, 28(4), 59-80.

Challacombe, F., \& Salkovskis, P. (2009). A preliminary investigation of the impact of maternal obsessive-compulsive disorder and panic disorder on parenting and children. Journal of Anxiety Disorders, 23, 848-857.

Chorpita, B. F., Brown, T. A., \& Barlow, D. H. (1998). Perceived control as a mediator of family environment in etiological models of childhood anxiety. Behavior Therapy, 29, 457-476.

Cobham, V. E., Dadds, M. R., \& Spence, S. H. (1999). Anxious children and their parents: what do they expect? Journal of Clinical Child Psychology, 28, 220-231.

Costa, N. M., \& Weems, C. F. (2005). Maternal and child anxiety: do attachment beliefs or children's perceptions of maternal control mediate their association? Social Development, 14, 574 590 . 
Dadds, M. R., Barrett, P. M., Rapee, R. M., \& Ryan, S. (1996). Family process and child anxiety and aggression: an observational analysis. Journal of Abnormal Child Psychology, 24, 715-734.

Dumas, J. E., LaFreniere, P. J., \& Serketich, W. J. (1995). "Balance of power": a transactional analysis of control in mother-child dyads involving socially competent, aggressive, and anxious children. Journal of Abnormal Psychology, 104, 104-113.

Gar, N. S., \& Hudson, J. L. (2008). An examination of the interactions between mothers and children with anxiety disorders. Behaviour Research and Therapy, 46, 1266-1274.

Gardner, F. (2000). Methodological issues in the direct observation of parent-child interaction: do observational findings reflect the natural behavior of participants? Clinical Child and Family Psychology Review, 3, 185-198.

Ginsburg, G. S., \& Schlossberg, M. C. (2002). Family-based treatment of childhood anxiety disorders. International Review of Psychiatry, 14, 143-154

Ginsburg, G. S., Grover, R. L., \& Ialongo, N. (2004a). Parenting behaviors among anxious and non-anxious mothers: relation with concurrent and long-term child outcomes. Child \& Family Behavior Therapy, 26, 23-41.

Ginsburg, G. S., Siqueland, L., Masia-Warner, C., \& Hedtke, K. A. (2004b). Anxiety disorders in children: family matters. Cognitive and Behavioral Practice, 11, 28-43.

Ginsburg, G. S., Grover, R. L., Cord, J. J., \& Ialongo, N. (2006). Observational measures of parenting in anxious and non-anxious mothers: does type of task matter? Journal of Clinical Child and Adolescent Psychology, 35, 323-328.

Gordon, D., Burge, D., Hammen, C., Adrian, C., Jaenicke, C., \& Hiroto, D. (1989). Observations of interactions of depressed women with their children. The American Journal of Psychiatry, 146, 50-55.

Greco, L. A., \& Morris, T. L. (2002). Paternal child-rearing style and child social anxiety: investigation of child perceptions and actual father behavior. Journal of Psychopathology and Behavioral Assessment, 24, 259-267.

Gueorguieva, R., \& Krystal, J. H. (2004). Move over ANOVA: progress in analyzing repeated-measures data and its reflection in papers published in the archives of general psychiatry. Archives of General Psychiatry, 61, 310-317.

Hawes, D. J., \& Dadds, M. R. (2006). Assessing parenting practices through parent-report and direct observation during parenttraining. Journal of Child and Family Studies, 15, 555-586.

Hudson, J. L., \& Rapee, R. M. (2001). Parent-child interactions and anxiety disorders: an observational study. Behaviour Research and Therapy, 39, 1411-1427.

Hudson, J. L., Doyle, A. M., \& Gar, N. (2009). Child and maternal influence on parenting behavior in clinically anxious children. Journal of Clinical Child and Adolescent Psychology, 38, 256-262.

Kendall, P. C., \& Chansky, T. E. (1991). Considering cognition in anxietydisordered children. Journal of Anxiety Disorders, 5, 167-185.

Keselman, H. J., Algina, J., \& Kowalchuk, R. K. (2001). The analysis of repeated measures designs: a review. The British Journal of Mathematical and Statistical Psychology, 54, 1-20.

Krain, A. L., \& Kendall, P. C. (2000). The role of parental emotional distress in parent report of child anxiety. Journal of Clinical Child Psychology, 29, 328-335.

Manassis, K., \& Bradley, S. J. (1994). The development of childhood anxiety disorders: toward an integrated model. Journal of Applied Developmental Psychology, 15, 345-366.

McCarty, C. A., Lau, A. S., Valeri, S. M., \& Weisz, J. R. (2004). Parent-child interactions in relation to critical and emotionally overinvolved expressed emotion (EE): is EE a proxy for behavior? Journal of Abnormal Child Psychology, 32, 83-93.
McLeod, B. D., Wood, J. J., \& Weisz, J. R. (2007). Examining the association between parenting and childhood anxiety: a metaanalysis. Clinical Psychology Review, 27, 155-172.

Moore, P. S., Whaley, S. E., \& Sigman, M. (2004). Interactions between mothers and children: impacts of maternal and child anxiety. Journal of Abnormal Psychology, 113, 471-476.

Rapee, R. M. (1997). Potential role of childrearing practices in the development of anxiety and depression. Clinical Psychology Review, 17, 47-67.

Rubin, K. H., \& Mills, R. S. (1991). Conceptualizing developmental pathways to internalizing disorders in childhood. Canadian Journal of Behavioural Science, 23, 300-317.

Shortt, A. L., Barrett, P. M., Dadds, M. R., \& Fox, T. L. (2001). The influence of family and experimental context on cognition in anxious children. Journal of Abnormal Child Psychology, 29, 585-598.

Silverman, W. K., \& Albano, A. M. (1996). The anxiety disorders interview schedule for children $(A D I S-C / P)$. San Antonio: Psychological Corporation.

Silverman, W. K., Saavedra, L. M., \& Pina, A. A. (2001). Test-retest reliability of anxiety symptoms and diagnosis with anxiety disorders interview schedule for DSM-IV: child and parent versions. Journal of the American Academy of Child and Adolescent Psychiatry, 40, 937-944.

Silverman, W. K., Kurtines, W. M., Pina, A. A., \& Jaccard, J. (2009). Directionality of change in youth anxiety treatment involving parents: an initial examination. Journal of Consulting and Clinical Psychology, 77, 474-485.

Siqueland, L., Kendall, P. C., \& Steinberg, L. (1996). Anxiety in children: perceived family environments and observed family interaction. Journal of Clinical Child Psychology, 25, 225-237.

Turner, S. M., Beidel, D. C., Roberson-Nay, R., \& Tervo, K. (2003). Parenting behaviors in parent with anxiety disorders. Behaviour Research and Therapy, 41, 541-554.

van der Bruggen, C. O., Stams, G. J. J. M., \& Bögels, S. M. (2008). Research review: the relation between child and parent anxiety and parental control: a meta-analytic review. Journal of Child Psychology and Psychiatry, 49, 1257-1269.

Varela, E. R., Vernberg, E. M., Sanchez-Sosa, J. J., Riveros, A., Mitchell, M., \& Mashunkashey, J. (2004). Anxiety reporting and culturally associated interpretation biases and cognitive schemas: a comparison of Mexican, Mexican American, and European American families. Journal of Clinical Child and Adolescent Psychology, 33, 237-247.

Vasey, M. W., Daleiden, E. L., Williams, L. L., \& Brown, L. M. (1995). Biased attention in childhood anxiety disorders: a preliminary study. Journal of Abnormal Child Psychology, 23, 267-279.

Weems, C. F., Zakem, A. H., Costa, N. M., Cannon, M. F., \& Watts, S. E. (2005). Physiological response and childhood anxiety: association with symptoms of anxiety disorders and cognitive bias. Journal of Clinical Child and Adolescent Psychology, 34, 712-723.

Whaley, S. E., Pinto, A., \& Sigman, M. (1999). Characterizing interactions between anxious mothers and their children. Journal of Consulting and Clinical Psychology, 67, 826-836.

Wood, J. J., McLeod, B. D., Sigman, M., Wei-Chin, H., \& Chu, B. C. (2003). Parenting and childhood anxiety: theory, empirical findings, and future directions. Journal of Child Psychology and Psychiatry, 44, 134-151.

Woodruff-Borden, J., Morrow, C., Bourland, S., \& Cambron, S. (2002). The behavior of anxious parents: examining mechanisms of transmission of anxiety from parent to child. Journal of Clinical Child and Adolescent Psychology, 21, 364-374. 